\title{
Extensive Craniofacial Fibrous Dysplasia: An Overview
}

\author{
Kavita Sachdeva
}

\section{ABSTRACT}

Introduction: Fibrous dysplasia (FD) is a common benign osseous tumor that may affect the craniofacial skeletal. It is a slowly progressing disease, presenting with swelling and pressure effects on adjacent structure. Epistaxis as presenting symptom is rare. The literatures reviewed rarely mention it.

Aim: The aim of this article is to study the clinical presentation and management of extensive craniofacial FD along with review of literature, emphasizing ear, nose, and throat/ endocrinal and orthopedic checkup and computer tomography evaluation and related hematological workup. Serum alkaline phosphatase was also done to see its correlation to disease activity. Brief overview of management and its related complication is done.

Results: Both patients were presented with 2-year history. There was no predisposing factor. Both were from tribal area. They were having slowly progressive nasal obstruction, facial swelling, and proptosis. In Case 1, it was loosening of tooth and facial pain. In Case 2, it was epistaxis which was the presenting complaint of patient. Nasal obstruction, swelling, and epistaxis in teenager male usually suggest angiofibroma. Craniofacial FD may have epistaxis and pain due to cystic degeneration in FD. Both patients had proptosis; however, their vision was normal. Serum alkaline phosphates was marginally raised, thus not signifying active disease. Computed tomography (CT) scan was diagnostic in both cases, with ground glass appearance. In Case 2, there was cystic degeneration present and CT was useful in diagnosing the disease, to show the extent of lesion and any intracranial extension. Fine needle aspiration cytology was inconclusive and incision biopsy is better to get representative sample. Lateral rhinotomy approach with lip splitting and gingival component gave good exposure. The tumor was totally removed with thin bone at periphery. The tumor was vascular, and bleeding was controlled by anterior nasal packing.

Keywords: Endocrinal, Fibrous dysplasia, Ground glass, Maxillary.

How to cite this article: Sachdeva K. Extensive Craniofacial Fibrous Dysplasia: An Overview. Clin Rhinol An Int J 2017;10(1):17-21.

\section{Source of support: Nil}

Conflict of interest: None

\section{Associate Professor}

Department of ENT, Netaji Subhash Chandra Bose Medical College \& Hospital, Jabalpur, Madhya Pradesh, India

Corresponding Author: Kavita Sachdeva, Associate Professor Department of ENT, Netaji Subhash Chandra Bose Medical College \& Hospital, Jabalpur, Madhya Pradesh, India, Phone: +07614004374, e-mail: drksachdeva@yahoo.co.in

\section{INTRODUCTION}

Fibrous dysplasia (FD) is a benign osseous condition in which normal bone and marrow are replaced by fibrous tissue and haphazardly distributed woven bone. ${ }^{1,2}$ The disease typically presents in the 1st and 2nd decade of life and then progresses slowly until patient reaches age of 30 .

Fibrous dysplasia can be in monostotic form affecting single anatomical area or polyostotic form in which multiple bones are involved. In McCune-Albright syndrome, it is defined by triad of polyostotic fibrous dysplasia (PFD) with skin pigmentation, premature sexual development, and hyperthyroidism. ${ }^{3}$ It is caused by somatic activation mutation of $G$ protein GMS gene.

Monostotic fibrous dysplasia (MFD) is the common manifestation of diseases. It is four times ${ }^{4}$ common than PFD, with location being craniofacial bone, proximal femur, and rib. ${ }^{2,5}$ In MFD, zygomatico maxillary complex is commonly involved. The symptoms for craniofacial MFD are facial deformity and asymmetry, vision change, hearing impairment, nasal congestion, and obstruction. Pain, paresthesia, and malocclusion are also reported.

This disease has slow indolent course when adjacent structures are affected. It presents as lacrimation, eye proptosis, seventh cranial nerve palsy, and nasal airway obstruction. In young children and adolescents, this lesion may cause rapid growth, cortical expansion, and displacement of adjacent bone, such as eye and teeth. Rapid growth may be associated with pathologies like aneurysmal bone cyst, mucoceles, or malignant transformation in $1 \%$ of the cases. ${ }^{6}$ After puberty, the lesion stabilizes. The complication occurs when lesions involve sphenoid, orbital apex, and frontal bones causing proptosis, visual disturbance, facial asymmetry, and orbital dysostosis.

\section{CASE REPORTS}

\section{Case 1}

A 32-year-old female, farmer by occupation from tribal area, reported to the ear, nose, and throat (ENT) outpatient department (OPD) with complaints of swelling in left maxillary area for the past 1 year and left nasal obstruction for 1 year. As per patient, she was apparently alright 2 years back when she developed increased lacrimation in left eye, which was insidious in onset, unilateral continuous, and got progressively worse 1 year back. She also noticed a nasal blockage and swelling in nasolabial 


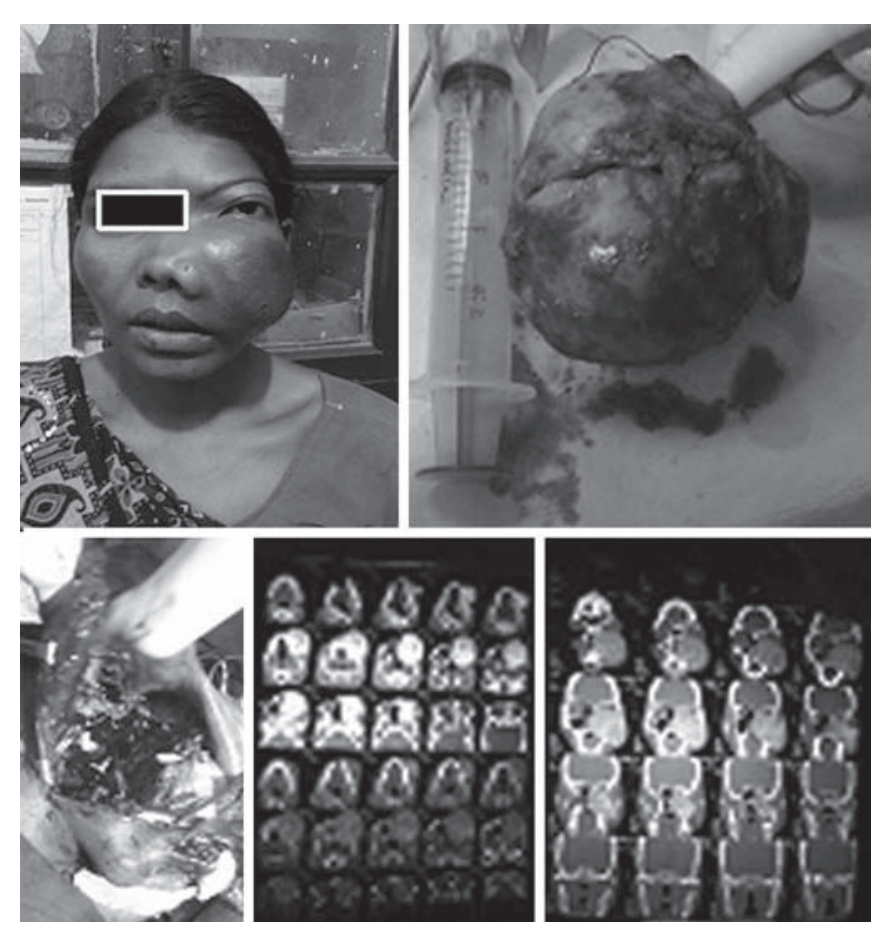

Fig. 1: Case 1

fold about size of a pea, which gradually increased to size of a tennis ball in 6 months. For the past 2 months, she has bilateral nasal obstruction and blurring of vision in the left eye. Since 2 weeks, she has pain, which is nonradiating along with progressive difficulty in chewing. Anosmia is also present. There is no history of epistaxis or dental extraction. She was addicted to tobacco chewing since 1 year. Menarche was at 11 years of age.

Patient applied some local herb on swelling with no relief, resulting in discoloration of premaxillary skin. There was no history of trauma and there was no other swelling on limbs or skull present and no signs of endocrinopathy.

On the left premaxilla, a huge globular swelling was present, measuring 10 to $12 \mathrm{~cm}$ in dimension. It extended from septum in midline, laterally extending in the infratemporal fossa up to zygoma. It further extended upward obliterating the infraorbital margin, pushing the eye upward, outward, and laterally inferiorly up to upper gingivobuccal sulcus. Nasolabial groove was obliterated. Margins were ill-defined. Overlying skin was darkly pigmented but was freely mobile and could be lifted freely from the deeper hard mass. No vasodilation or bruit was present. On palpation, this hard globular swelling was nontender and nonpulsatile. No signs of inflammation were present. Slight pain was present on deep palpation.

Perorally, mouth opening was adequate. Swelling of hard palate on left was present, pushing it downward with loosening of left premolar tooth.

Pernasally, a mass was present in left nasal cavity, blocking it completely arising from left lateral wall pushing septum to right side touching right lateral wall of nose blocking both nostrils. It was hard in consistency. Probe cannot be passed all around. No ulceration present in mucosa; no bleeding present; air entry bilaterally absent. On postrhinoscopy, no mass present in nasopharynx and no other swelling present on skull or body.

Exophthalmos and proptosis of left eye present along with telecanthus vision; fundus examination was normal; no lymphadenopathy present.

Lab investigations showed mild anemia with hemoglobin $9.9 \mathrm{gm} / \mathrm{dL}$ and mildly raised serum alkaline phosphatase levels. All other hematologic and biochemical parameters were normal. Chest X-ray and X-ray of limbs were normal.

Fine needle aspiration cytology (FNAC) revealed only mucoid aspirate, which was inconclusive. Conventional white light (CWL) biopsy done, which reported a benign spindle cell lesion that lacked pleomorphism, giant cells, and atypia. Calcified curvilinear fragments of osteoid tissue were present throughout and were not rimmed by osteoblasts. These features were consistent with FD.

Computed tomography (CT) scan showed a large, well-delineated, expansile bony lesion with ground glass appearance (fibrous and ossific mixture) arising from within left maxilla causing indentation and remodeling of left orbital floor superiorly, left half of hard palate inferiorly, and nasal septum medially. It measures $7.6 \times$ $6.4 \times 7 \mathrm{~cm}$ in size. Extension of lesion in left infratemporal fossa posteriorly and soft tissue of cheek outwardly is seen. Left sphenoid, ethmoid sinuses show opacification.

Left-side orbit proptosis is seen showing compression by expansile lesion from inferior side. Bilateral nasal cavity is occluded, suggestive of a benign looking welldefined expansile fibro-osseous neoplastic lesion of left maxilla with extension.

Management of surgical excision of mass was done. Weber Ferguson incision was given; outer wall of maxilla was thinned. On removing the wall, the globular mass was seen. The mass was dissected all around. It was causing expansion of maxilla posteriorly; superiorly orbital wall was paper thin; inferiorly tooth root were loose along with right-sided shifting of septum. The mass removed was hard causing expansion of maxillary bone. Bleeding was present which was controlled by packing. Manual compression of septum done and both nasal patency restored. Histopathology report was consistent with FD. Postoperative recovery was uneventful; proptosis started decreasing; and both nasal cavity were patent.

\section{Case 2}

A 16-year-old male student from rural area came to ENT OPD, with history of (h/o) nasal obstruction for 3 years; nasal bleed for 2 years. 

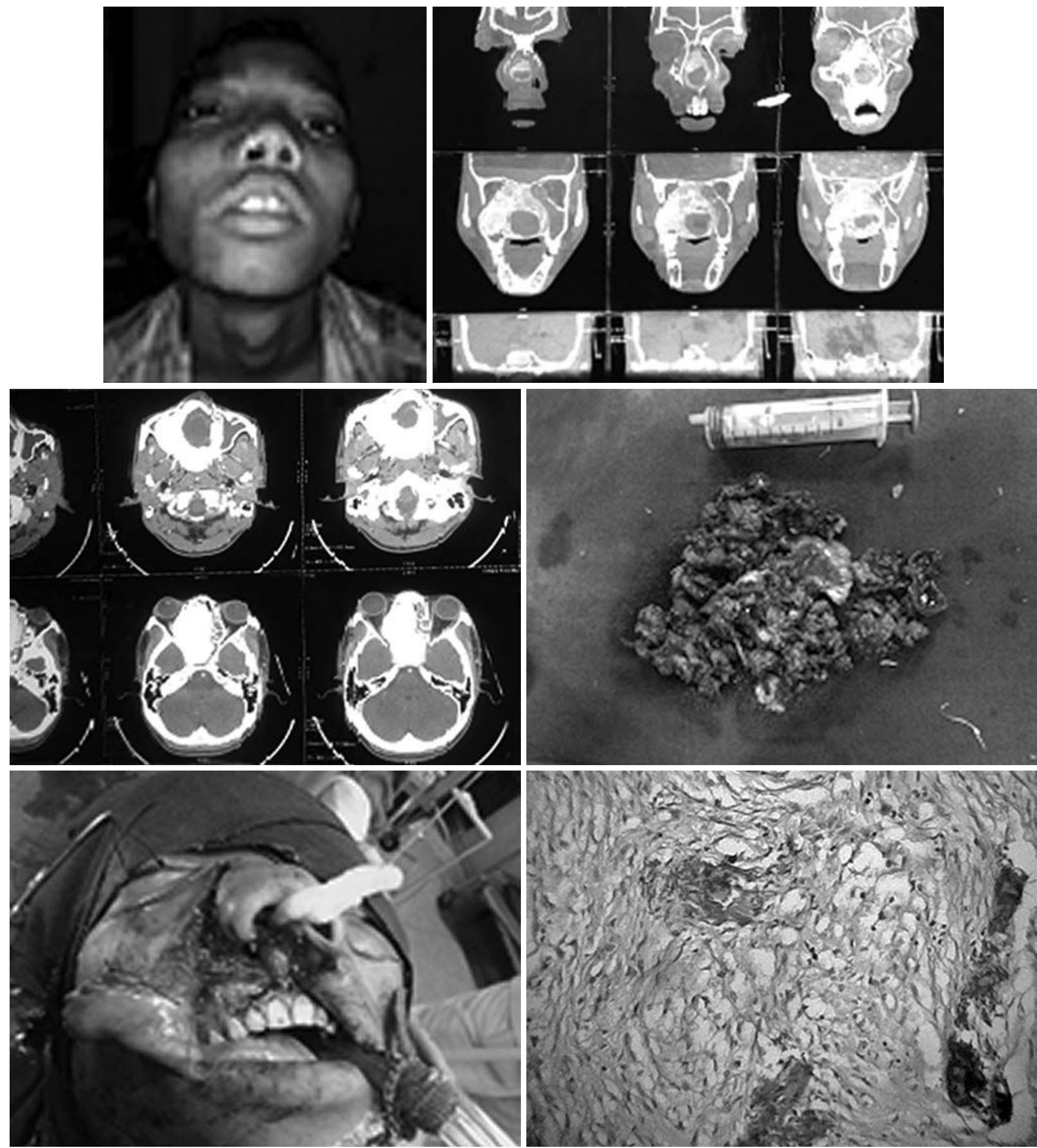

Fig. 2: Case 2

The patient was apparently alright 3 years back when he started experiencing right-sided nasal obstruction. Gradually it became bilateral. Since past 2 years, he is mouth breather and also having episodes of right-sided nasal bleeding, spontaneous and significant. Frequency of epistaxis has increased, hyposmia, facial deformity (zygoma), and hyponasal voice were present.

History of jaundice 6 months back; no h/o of trauma or paresthesia of cheek present. There is no diplopia; no $\mathrm{h} / \mathrm{o}$ weight loss; he is a chronic smoker; occasional alcohol consumer; and was previously addicted to ganja.

Systemic examinations were normal. Local examination revealed a huge swelling involving right half of face extending from right lateral margin of nose to zygoma superiorly displacing eye upward and outward causing proptosis, and hypertelorism zygoma is prominent.

On pernasal examination, a pinkish polypoidal mass lesion was present in right nostril completely filling 
it, firm in consistency. Mucosa overlying is inflamed. Septum has been pushed to opposite side by the mass and is abutting the left lateral wall of nose. Purulent discharge was present in nasal floor. Probe could not be passed around it as it is sensitive to touch. Submandibular lymph node palpable. Air entry in both nose absent.

Postrhinoscopy, no mass lesion seen in nasopharynx. Infraorbital sensation was normal. On peroral examination, bulge of hard palate and gingivobuccal sulcus fullness present on right side. Vision and fundus were within normal limit (WNL). Hypertelorism and axial proptosis of right eye present.

Computed tomography scan showed right nasal, maxillary, and ethmoid cavities were expanded. These spaces as well as sphenoid show peripherally calcified, almost ossified lesion with soft tissue in the center of this lesion, causing bulge of right retro-orbital fossa and left maxillary sinus, which was also suggestive of FD.

Hemoglobin $8.5 \mathrm{gm} \%$, platelet adequate, serum alkaline phosphatase was $45 \mathrm{IU}$, rest hematological tests were WNL, and patient international normalized ratio was 1.2. His FNAC was reported as hemorrhagic aspirate.

Punch biopsy was inflammatory lesion with bone bits and mononuclear infiltrate. Surgery done by Weber Ferguson incision. Dysplastic tissue was removed piecemeal. It was going up to sphenoid, postethmoid, orbital apex, and superiorly up to skull base. Outer thin cortex was present delineating it from normal adjacent bone. There was bleeding from central necrotic area (preoperatively three points of blood was given).

Postoperative recovery was uneventful, except patient complaining of mild retro-orbital pain. Vision was normal.

\section{DISCUSSION}

The MFD in India is not much common. Case reports are mostly from tertiary referral center. The true incidence is not documented. There is no sex predisposition; age of presentation is 18.3 years. The MFD is a common manifestation of FD disease. The most common location is craniofacial bone $(90 \%)$ zygomatic maxillary complex. $^{3}$ Sinuses may be affected commonly and are sphenoid than ethnoid and maxillary sinus. Patients have nasal blockage, hyposmia facial pain, lacrimation, and loosening of tooth. The alveolar arches are well maintained. In eye proptosis, dystopia and hypertelorism may occur. Nasolacrimal duct obstruction, eye closure problem, or trigeminal neuralgia may also occur. The temporal bone may be involved in $70 \%$ cases. ${ }^{7}$ The two cases presented here incidentally came on the same day to our OPD and were admitted with probable diagnosis of myxoid chondroma (Case 1) and angiofibroma (Case 2) under evaluation. Case 1 presented with bilateral nasal obstruction, pain in tooth, loosening, facial discoloration, and proptosis. Case 2 presented with epistaxis and nasal obstruction.

\section{DISCUSSION}

A thorough clinical examination is done to know the extent of lesion, whether MFD or PFD. History of menarche, endocrinal examination, and abnormal pigmentation should be evaluated.

Common radiographic finding of ground glass appearance with thinned cortex without distinct border ${ }^{5}$ is suggestive of FD. It may vary from ground glass to mixed radiodense and radiolucent area.

Computed tomography scan is mandatory in the evaluation of craniofacial FD. Ill-defined margin of lesion helped to differentiate it from other fibro-osseous lesion. The characteristic CT picture may be pagetoid, sclerotic, and cystic. Pagetoid describes a mixture of dense and radiolucent area of fibrosis, sclerotic lesion are homogeneously dense, and cystic as ovoid radiolucency with dense periphery. Cystic degenerative is uncommon, described occasionally in the literature. Pagetoid with expansion of cortex is common in $55 \%$, cystic $11 \%$, and sclerotic $34 \% .{ }^{8}$ Magnetic resonance imaging (MRI) is required in cystic degeneration and malignant degeneration of FD and for differentiating cyst from mucocele.

Macroscopy of FD revealed expansion of osseous trabecula into a thin cortical. There is no capsule. Microscopy shows collagen matrix stoma with fibroblast in entangled strand with osseous trabeculae. These irregular trabeculae have Chinese letter pattern. Biopsy is important to confirm diagnosis. Nonepithelial lining of cyst of FD varies in nature, and it can be aneurysmal bone cyst or simple bone cyst. The cystic degeneration is associated with acute clinical changes.

The FD differential diagnosis includes benign lesions, ossifying fibroma, Paget's disease, aneurysmal bone cyst, ameloblastoma, osteochondroma, and malignancies like sarcoma and metastatic osteoblastic lesion., ${ }^{9,10}$

Management of clinical examination and radiograph help in making diagnosis. Both patients after workup having provisional diagnosis of MFD were operated. In case 1, the tumor was removed as single mass. However, in case 2 cysts degenerated, so it was removed piecemeal, extending up to past ethmoid and sphenoid sinus.

Surgery for FD should be postponed until adolescence or until indicated for cosmetic appearance. Asymmetry and swelling are common complaints requiring surgery. Evaluation was done by CT scan and biopsy. If asymptomatic and small, periodic follow-up is recommended; 
however, for symptomatic and associated functional defect or cystic change associated with sudden increase in size and if vision is compromised, a repeat CT scan evaluation and aggressive management is recommended. For degenerated lesion, management according to stage of activity is warranted. In quiescent stage close follow-up or surgical contouring is advised, and if resectable complete excision. In nonaggressive stage wait till it becomes quiescent and patient has reached skeletal maturity. Patient with aggressive and rapid FD with pain, paresthesia, or nasal obstruction, after proper evaluation by CT scan should be evaluated for aneurysmal bone cyst. If malignant changes or mucocele may be seen then surgery may be contouring or en bloc resection. If conservative resection is done, there is risk of recurrence. Moreover, FD is vascular and bleeds.

Osteomyelitis should be treated with antibiotic. Nonsurgical management include bisphosphonates, such as pamidronate and alendronate to reduce rate of growth and pain. The response to these agents is assessed by decrease in pain and decreasing value of serum alkaline phosphatase.

Common neurological complication is blindness, hence in patients $\mathrm{CT}$ evaluation on cyst compressing optic nerve is seen and immediate decompression and resection is recommended. Malignant transformation may rarely occur in less than $1 \%$ of FD.

\section{RESULTS}

Both patients were presented with 2-year history. There was no predisposing factor. Both were from tribal area. They were having slowly progressive nasal obstruction, facial swelling, and proptosis. In Case 1, it was loosening of tooth and facial pain. In case 2, patient presented with complaints of epistaxis and nasal obstruction, suggesting angiofibroma. The patient may have epistaxis and pain along with cystic degeneration in FD. Both patients had proptosis; however, their vision was normal.

Serum alkaline phosphate was marginally raised, thus indicating nonaggressive stage. In both, CT scan was diagnostic with ground glass appearance and in Case 2, there was cystic degeneration present. The CT scan was useful in diagnosing disease and to show extent of lesion as well as to rule out any intracranial lesion. The FNAC of lesion was not of much help. In FD of maxilla, CWL biopsy or incision biopsy is better to get a representative sample. Lateral rhinotomy approach with lip splitting and gingival component gave good exposure. The tumor was totally removed with thin bone at periphery. The tumor was vascular and bleeding was encountered, which required simple anterior nasal packing. On the second postoperative day, the patient had good airway and good vision with good cosmetic result. By the end of 15th day, proptosis started decreasing. No residual growth was present in follow-up.

\section{CONCLUSION}

Fibrous dysplasia is one of the most common benign osseous craniofacial tumors. Normal medullary bone is gradually replaced by abnormal fibrous connective tissue. It presents with facial swelling, nasal blockage, and rarely with epistaxis. Sinuses may be affected and are commonly sphenoid than ethnoid or maxillary sinus. Patients have nasal blockage, hyposmia, facial pain, lacrimation, and loosening of tooth. Functional endoscopic sinus surgery has no role in treatment.

Cystic degeneration may cause sudden acute symptom like epistaxis or blindness by compressing optic nerve.

\section{REFERENCES}

1. Lee JS, FitzGibbon EJ, Chen YR, Kim HJ, Lustig LR, Akintoye SO, Collins MT, Kaban LB. Clinical guidelines for the management of craniofacial fibrous dysplasia. Orphanet J Rare Dis 2012 May 24;7 (Suppl 1):S2.

2. Riminucci M, Fisher LW, Shenker A, Spiegel AM, Bianco P, Gehron Robey P. Fibrous Dysplasia of bone in the McCuneAlbright syndrome: abnormalities in bone formation. Am J Pathology 1997 Dec;151(6):1587-1600.

3. Riminucci M, Liu B, Corsi A, Shenker A, Spiegel AM, Robey PG, Bianco P. The histopathology of fibrous dysplasia of bone in patients with activating mutations of the Gs alpha gene; site-specific patterns and recurrent histological hallmarks. J Pathol 1999 Jan;187(2);249-258.

4. Parekh SG, Donthineni-Rao R, Ricchetti E, Lackman RD. Fibrous dysplasia. J Am Acad Orthop Surg 2004 Sep-Oct;12(5): 305-313.

5. Chen YR, Wong FH, Hsueh C, Lo LJ. Computed tomography characteristics of non-syndromic craniofacial fibrous dysplasia. Chang Gung Med J 2002 Jan;25(1):1-8.

6. Sadeghi SM, Hosseini SN. Spontaneous conversion of fibrous dysplasia into osteosarcoma. J Craniofac Surg 2011 May:22(3):959-961.

7. Pouwels AB, Cremers CW. Fibrous dysplasia of the temporal bone. J Laryngol Otol 1988 Feb;102(2):171-172.

8. Diah E, Morris DE, Lo LJ, Chen YR. Cyst degeneration in craniofacial fibrous dysplasia: clinical presentation and management. J Neurosurg 2007 Sep;107(3):504-508.

9. Eller R, Sillers M. Common fibro-osseous lesions of the paranasal sinuses. Otolaryngol Clin North Am 2006 Jun;39(3):585-600.

10. Edgerton MT, Persing JA, Jane JA. The surgical treatment of fibrous dysplasia. With emphasis on recent contributions from cranio-maxillofacial surgery. Ann Surg 1985 Oct;202(4): 459-479. 